\title{
Cómo afrontar el diseño editorial en la difusión y promoción de la cultura artística en Bellas Artes. Proyecto de innovación docente en la asignatura de "Arte, Diseño y Comunicación"
}

\begin{abstract}
How to deal with editorial design in the dissemination and promotion of artistic culture in Fine Arts. An innovative proposal to teach the subject "Art, Design and Communication"
\end{abstract}

MANUEL FERnANdo MANCERA MARTÍNEZ

ORCID: https://orcid.org/0000-0001-7950-8859

Universidad de Sevilla

Departamento de Dibujo

mancera@us.es

Fecha de recepción: 23-05-2019.

Fecha de aceptación: 28-05-2019.

DOI: http://dx.doi.org/10.12795/9788447221912.005

Pр.: 132-152 


\section{Resumen}

En el presente artículo vamos a abordar la experiencia de una innovación docente en relación al diseño editorial en la difusión y promoción de la cultura artística. Nos basaremos en la eficiencia y la calidad como gestoras dentro del programa de la asignatura Arte, Diseño y Comunicación del Grado en Bellas Artes. La innovación llevada a la práctica se ha fundamentado en un nuevo enfoque metodológico, próximo a los planteamientos del Aprendizaje Basado en Proyectos y el Design Thinking. Estos pueden mejorar la gestión y el uso de las distintas herramientas desarrolladas dentro del ámbito educativo, de manera genérica, y del diseño de manera focalizada en esta materia. Se ha apostado por empoderar al alumnado como elemento determinante para indagar en el proceso intelectual, asimilando los conceptos desde la praxis y testeando los resultados para profesionalizar los distintos productos. La experiencia ha resultado muy satisfactoria en ambas direcciones, aportando mayor participación y madurez.

Palabras clave: Arte, Diseño y Comunicación; Bellas Artes; docencia universitaria; experimentación docente universitaria; difusión y promoción de la cultura artística.

\section{Abstract}

In this article we'll improve a teaching innovation experience in relationship with the editorial design in the diffusion and promotion of the artistic culture. We will be based on efficiency and quality as managers within the program of the subject Art, Design and Communication of the Degree in Fine Arts. The innovation put into practice has been based on a new methodological approach, close to the approaches of Project-Based Learning and Design Thinking. These one can improve the management and use of the different tools developed within the educational field, in a general way, and particularly in this matter. It has opted to empower students as a determining factor to investigate the intellectual process, assimilating the concepts from practice and testing the results to professionalize the different products. The experience has been very satisfactory in both ways, providing greater participation and maturity.

Keywords: Art, Design and Communication; Fine arts; University teaching; university teaching experimentation; dissemination and promotion of artistic culture. 


\section{Descripción del contexto}

Arte, Diseño y Comunicación es una asignatura optativa de cuarto de carrera de Grado en Bellas Artes de la Universidad de Sevilla. En ella se han aplicado los dos ciclos de mejora en el aula promovidos por el Programa de Formación e Innovación Docente del Profesorado (FIDOP), coincidiendo con el segundo cuatrimestre del curso 2018/19. La asignatura en su totalidad es impartida por el mismo profesor, y los grupos están formados por 27 alumnos, como números clausus. No obstante, existe una alta demanda fruto de la buena acogida que ha tenido en años anteriores. Por ello la asignatura se ha ido revalorizando, siendo considerada como una disciplina necesaria para desenvolverse y mostrarse al público, desde un prisma profesional y competitivo.

\section{Diseño previo del CIMA}

\section{Mapa de contenidos}

Si bien la asignatura se construye en torno a tres bloques temáticos generales, la idea de diseñar un proyecto final global hace que los compartimentos estancos de los mismos queden abiertos, y se interactúe arbitrariamente según lo vaya necesitando el proyecto escogido. Por ello, el segundo ciclo de mejora no fue implementado en un bloque temático concreto, sino que, se apoyó en la idea genérica del tema principal, el cual aborda la esencia de cualquier proyecto que se pueda proponer en el aula: el diseño editorial en la difusión y promoción de la cultura artística.

De este modo, cualquier propuesta o intención de desarrollo, siempre se verá consolidada por la identidad del 
concepto maqueta y tratamiento editorial, reforzando en todo momento el valor de construir desde la máxima calidad. Es por ello que se tiene el máximo control en los distintos parámetros de salida, según las necesidades finales de nuestro producto.

Así pues, se elaboró un mapa de contenidos (ver Figura 1) en el que mostrar el sistema editorial de divulgación. En él se verían implicados todos y cada uno de los distintos bloques temáticos del programa docente (Gómez-Aguilar, 2018).

A la hora de avanzar en el proceso de mejora se identificaron algunos problemas relativos a las dificultades a nivel procedimental y actitudinal del alumnado para reconocer los problemas técnicos iniciales. Con este segundo ciclo de mejora se ha buscado pasar de la abstracción del concepto a la búsqueda de ese concepto en ejemplos reales. Se consigue con ello que el proceso intelectual que deviene de la praxis esté a priori identificado en un problema ya resuelto. De ese modo, se valida visualmente, siendo comprendido el protocolo más rápidamente, al ser un referente para el proyecto desarrollado en el aula como, por ejemplo, determinar la composición de la página y/o el pliego, de manera que el contenido se ajuste convenientemente a los requisitos mínimos que debe cumplir.

Jornadas de Formación e Innovación Docente del Profesorado | № 2 (2019) Esta obra se distribuye con la licencia Creative Commons 


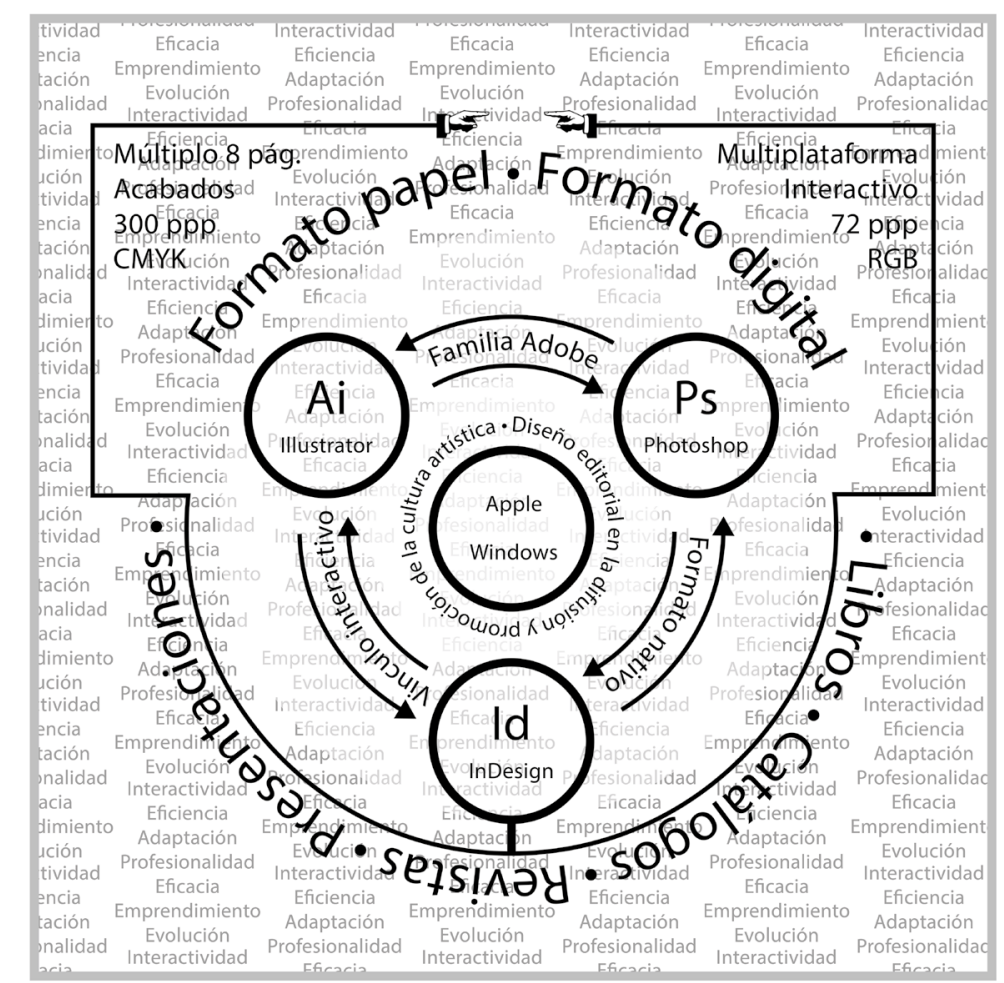

Conceptuales

Figura 1. Mapa de contenidos del segundo ciclo de mejora en el aula

\section{Implicaciones del modelo metodológico posible para el diseño de la secuencia de actividades}

A continuación, se muestra el modelo metodológico (Figura 2) diseñado, en el que se constata la retroalimentación de datos, opiniones y propuestas diversas que enriquecen y mejoran la totalidad de la práctica.

La asignatura desde hace años se centra en un desarrollo teórico-práctico que afronta los distintos retos que se plantean mediante proyectos como, por ejemplo, la maquetación de una revista o de una publicación seriada. 
Por inquietudes personales, esta asignatura ha ido evolucionando poco a poco. Se ha apostado por no abordar la enseñanza de los distintos contenidos a través de meros ejercicios aislados para aprender distintas herramientas y conceptos. A lo largo de varios años se ha ido construyendo, desde un proceso profesional, el desarrollo de la materia. Con ello se consigue abordar los distintos problemas y sus posibles soluciones según la demanda del mercado actual. Se aplican en los mismos los intereses sociales sobre los que hay que validar los distintos productos y testear los diferentes resultados.

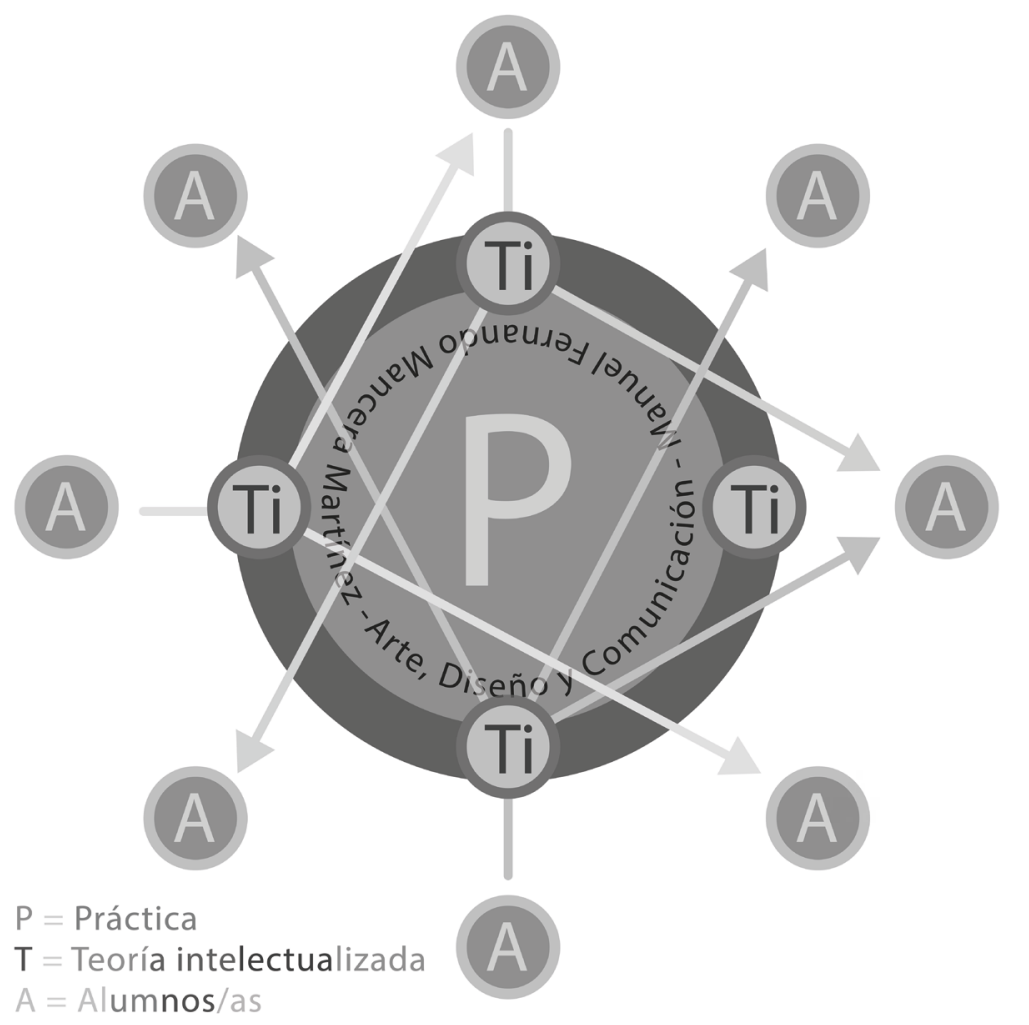

Figura 2. Modelo metodológico deseable.

Para ello, a lo largo de los años en la asignatura se han ido cambiando pautas y modelos de gestión. Desde 
un principio la materia ha estado dividida en tres grandes bloques, a los que van asociados tres softwares específicos de adobe: InDesign, Illustrator y Photoshop. Esto hacía que el temario quedase aislado por contenidos estancos; distribuir contenidos de manera abierta por proyectos fue la solución. De este modo podemos gestionar la materia de manera más atractiva, menos monótona y más interactiva, al ir trabajando conjuntamente un mismo proyecto con la aplicación de cada uno de sus contenidos pertinentes.

Entre otros proyectos que se van actualizando cada año a demanda de intereses sociales y personales, uno de esos "ejercicios" (proyectos) que quedan propuestos en el propio programa de la asignatura, es el diseño y maquetación del TFG. Este modo de afrontar un reto en el aula como proyecto real se sustenta en el aprendizaje basado en proyectos (Vergara Ramírez, 2016), ampliamente conocido en la Facultad de Bellas Artes de Sevilla desde hace muchos años. La experiencia previa ha demostrado que, además de eficaz, involucra al colectivo educativo a empatizar con los distintos problemas que se pueden plantear a lo largo del proceso.

El primer punto de conexión con la innovación radica en promover y difundir la propia materia desde los modelos técnicos y estéticos que se van a promover en la materia. Prescindiendo de los obsoletos .pptx, .mp4, etc. y apostando por pdf interactivos a pantalla completa con interacción integrada y diseñadas gráficamente para hacerlas atractivas y actuales, según las demandas sociales imperantes. Todo ello ha ido unido a sondeos puntuales que van indagando en los distintos intereses que puedan identificarse en grupos diversos. Dichos análisis se procesan a diario al comienzo de las clases consultando inquietudes, valoraciones personales, gustos e, incluso, sensaciones que se exponen en común y hacen que el colectivo interactúe y extraiga conclusiones de valor, para avanzar y mejorar intelectual y profesionalmente. 
Toda la teoría referente al proceso constructivo del modelo editorial ha sido gestionada en base a preguntas que, de manera inclusiva en la realidad de productos ya existentes en el mercado, los llevarán a la deducción de las herramientas a utilizar.

La interacción y la implicación del alumnado por reconocer y, con ello, sentirse capaces de formular propuestas en relación a los distintos parámetros técnico-creativos en los ejemplos que nos presenta el mercado editorial en la actualidad, ha promovido un compromiso y una dedicación mayor que en años anteriores. La captación de la teoría mediante identificación en los productos reales (Swartz, Costa, Beyer, Reagan y Kallick, 2016) ha sido todo un logro para avanzar en la proyección personal del alumnado.

La libertad a la hora de plantear los distintos contenidos en un modelo abierto, que sea atendido en momentos puntuales por las distintas necesidades que vaya demandando el proyecto, hacen que las actividades puedan resolverse de modo más ameno; con ello se suscita una mayor dedicación e interés respecto a anteriores tentativas.

Una vez se han detectado estas acciones se desarrollan las ocho sesiones del ciclo de mejora con el fin de identificar el contenido a trabajar en el mapa y adecuarlo de manera programada a las distintas opciones técnicas y estéticas que se puedan producir bajo demanda: 1.- elección de formato editorial; 2.- composición; 3.- tratamiento de recursos; 4.- aplicación de contenidos; 5.- tablas de contenidos dedicadas; 6 .- exportado de formatos multiplataforma; 7.- empaquetado.

A lo largo del cuatrimestre se van dando puntualmente datos relativos a las necesidades, cualidades y requisitos que debe contener un producto de calidad que sale al mercado editorial, con independencia del nicho de mercado consultado y del formato final. Para ello, se añaden al 
discurso intelectual que debe perseguir todo profesional de la edición, una serie de ejemplos base que demarcan la singularidad de un trabajo en distintos niveles de calidad y profesionalización. Con ello se aclaran y determinan los pilares fundamentales en los que se enmarca el tema a tratar en el ciclo de mejora.

Dado que el material a procesar queda abierto por el planteamiento inicialmente expuesto, las distintas sesiones se han enfocado a comprender de manera genérica, libre y abierta, las diversas posibilidades de actuación en un producto editorial, según las propuestas personales que han decidido construir como modelo.

Cabe destacar que la dinamización del aula es mucho más agotadora y también más gratificante al estar siempre en ebullición y constatar que el alumnado, cuando se le oferta algo que le suscita interés -y a lo que le pueden sacar partido en un futuro-, se implica más. Es una tarea más alentadora y satisfactoria e inyecta positividad general en el colectivo.

\section{Cuestionario inicial-final}

Se ha realizado un sondeo a través de un cuestionario, el cual ha perseguido el reconocer las posibles lagunas, que dentro del protocolo de edición existen, y sin las cuales un proyecto de la envergadura que se propone en el cuatrimestre no tendría viabilidad. Los resultados arrojados, que analizaremos más adelante, nos alertan respecto al sondeo previo que debemos promover para construir desde la certeza.

En un principio el diseño de este cuestionario no ha sido el más interesante una vez concluido todo el ciclo. En cierto modo ahora se elaboraría de distinta manera. No obstante, el resultado ha sido satisfactorio y ha servido 
para sondear y conocer los distintos problemas y cuestiones que afronta el alumnado al no procesar bien los conceptos. En torno a cinco preguntas encadenadas se ha procesado el modelo lógico en el diseño editorial.

1. ¿Es lo mismo editar que publicar? Razona tu respuesta.

2. ¿Existen diferencias entre productos del mundo editorial cultural y otro tipo de ediciones o publicaciones?

3. Dos diseñadores trabajan para una misma editorial - magazine: D1 está acostumbrado a guardar toda la documentación referida a cada número del magazine en una carpeta raíz específica subdividida por grandes grupos. D2 sin embargo en su método almacena la información que le llega a veces en la carpeta descarga, otras en alguna carpeta personal sin orden ni concierto e incluso hace cambios en cualquier momento del proceso. Explica si existe algún problema a la hora de maquetar el número de la revista que proceda y por qué.

4. ¿Qué aspectos debemos tener en cuenta para construir una maquetación?

5. Te encargan hacer una revista que, te dicen, en principio, va a salir en formato digital. ¿Qué hay que tener en cuenta a la hora de editar o maquetar esa revista?

A veces damos por sentados ciertos conceptos básicos que, sin embargo, por los datos contrastados de la encuesta, vemos que no son identificados como tales. Esto ha ayudado a formular nuevas mejoras futuras y a afrontar el último tramo del cuatrimestre con unos identificadores puntuales para asentar mejor los datos, bastante más positivos, arrojados con la encuesta final. 


\section{Aplicación del CIMA}

El ciclo de mejora se ha desarrollado a lo largo de ocho clases (todas en sesiones de dos horas), tanto en el grupo de mañana como en el de tarde con pequeñas variantes, que quedan resumidas de la siguiente manera:

\section{Primera sesión}

El comienzo de las clases siempre es igual, empatizando e interesándonos por cuestiones propias del diseño y de la actualidad del ámbito artístico desde el prisma de cada uno de los estudiantes. En esta ocasión se informa al colectivo, antes de empezar el proyecto de edición para la difusión y promoción de la cultura artística, de que se les pasará una encuesta (anónima en caso de desearlo) por encontrarnos inmersos en el Programa de Formación e Innovación Docente del Profesorado 2019. En el grupo de mañana asistieron ese día 22 alumnos y en el de la tarde 25. Algo interesante, ya que las preguntas estaban directamente relacionadas con el proyecto a afrontar, fue hacer grupos que determinaran y analizaran las respuestas dadas, exponiendo de manera colectiva un resultado consensuado, que reforzaría parte del trabajo a desempeñar a posteriori.

Para identificar los distintos tipos de modelos editoriales y publicaciones existentes en el mercado se les insta a buscar en plataformas digitales facilitándoles, además, distintos formatos físicos de revistas y libros. De esta manera podrían ver en ejemplos reales los dos posibles modelos básicos que trabaja la publicación de material editorial.

Algo muy llamativo es que, cuando buscan contenido, lo hacen según sus gustos personales. Esto, que es un condicionante restrictivo, por quedar relegada la búsqueda 
a conocimientos previos, finalmente mejora al promover un punto de vista que pivota sobre sus creencias y se les muestra algo realmente competitivo, llamativo, diferente, actual y les encanta. No obstante, mientras que no se les advierte de ello, no son capaces de buscar por palabras clave para localizar cualquier material referente al tema, si no que relegan la búsqueda a lo poco que conocen.

Analizando y proyectando las herramientas adquiridas hasta el momento, es ahora cuando se les da forma, circunscribiendo las posibilidades de trabajo y las necesidades relativas a la construcción del proyecto. Tamaño, relación de medidas, composición de pliego..., cualidades del proyecto en definitiva que harán que, según vayamos implementando contenido (tanto en InDesign como vinculando externamente desde Illustrator o Photoshop), todo esté correctamente gestionado y no genere problemas ulteriores.

El inicio del proyecto, buscando la personalización y el gusto personal, se centra en una composición "abierta", que sirva para una publicación "tipo" (según los modelos identificados y extraídos en la indagación anterior), de modo que pueda ser utilizado (con pequeños cambios si fuera necesario) tanto para su Trabajo Fin de Grado, como para catálogos u otros proyectos propuestos a principio de curso.

Como lo primordial en este proceso es saber sintetizar la idea y conseguir abocetar la composición en cuanto a espacios, ocupación y gestión de color, entre otras, se les mostraron elementos simples (acordes al modelo gestor del propio software constructor), y así pudieron desarrollar con agrado las ideas que se les iban ocurriendo.

Para propiciar el proceso intelectual, el docente se "entorpece" con la intención de que el alumnado sea quien diga: "cuidado, que si lo haces así va a dar problemas" o "¿pero lo has hecho en la capa que le corresponde?". 
Fue gratificante saber que el primer ciclo de mejora había dado sus frutos. Dominaban los conceptos básicos abordados y eran capaces de reconocer mínimos de calidad. No obstante, frente a ciertas inquietudes, se les insta a pensar: "¿qué te gustaría ver en una publicación que nunca has visto?". Identificar el "problema" como un posible deseo hecho realidad les resulta interesante y se implican más en querer saber si sería mejor un modo u otro de componer el pliego.

\section{Segunda sesión}

Como ya hemos indicado, los viernes están dedicados a afrontar el proyecto desde la gráfica con el software Illustrator. Por ello, se toma como referente el procedimiento aplicado el día anterior, pero identificando y procesando intelectualmente, en esta ocasión, los gráficos destacables dentro de productos existentes en el mercado.

Indagamos por tanto en el modo de procesar los gráficos de manera eficiente para, en cualquier momento, poder implementarlo ("colocar", como se identifica en la familia Adobe) según nuestras preferencias. Se trabajan, como ejemplos, simulaciones de autores de prestigio.

El sólo hecho de conseguir desarrollar un gráfico, con la misma calidad que algún creativo o ilustrador famoso, les llena de satisfacción y orgullo, sobre todo cuando se les oye decir: "esta herramienta es la bomba" (suavizado el término); o "con esto me hago yo ya lo que se me antoje". No obstante, hay muchas preguntas que quieren, inquietos, resolver con un chasquido de dedos, ante lo que se les espeta...: "eso para el máster de Diseño Gráfico" ya que no son conscientes aún de que, más que avanzar conociendo herramientas, lo interesante es dominar profesionalmente los mínimos indispensables para ser competitivos en el mercado laboral (siendo una lástima que haya desaparecido la especialidad que en el plan 80 había de Diseño Gráfico y habiendo sido relegada a esta única asignatura optativa de cuarto). 


\section{Tercera sesión}

Como en todas las sesiones, al inicio de las mismas se les sondea buscando inquietudes y así poder testear el proyecto a distintos niveles.

Personalmente me resulta inquietante que su ancho de miras, como hemos comentado ya anteriormente, quede relegado a lo poco que conocen o aquello que les gusta, $y$ que, sin embargo, cuando se les ofertan nuevos elementos los reciben con verdadera pasión. Como reto profesional está siempre presente el alentarlos con contenido nuevo, actualizado, competitivo, en definitiva, ilusionante.

La propia teoría referente a la gestión de contenido y el uso de herramientas específicas para desarrollarlas suele ser un problema, ya que el concepto es demasiado abstracto y cuesta identificar en los referentes el proceso hasta que se trabaja (cosa que no sucede en esta sesión). Se proponen distintas fórmulas para ver cómo dominar los distintos contenidos que puede contener una publicación. Aunque al principio puede ser engorroso y el alumnado hace amagos de derrumbarse y expresar su frustración, con dos o tres intervenciones personalizadas en su propio trabajo es más fácil de lo que se aprecia al principio. Pero volvemos a lo anteriormente expuesto, lo ven, pero a los dos minutos vuelven a caer en la misma problemática, porque sólo han simulado lo que se les ha dicho, pero no lo han comprendido y asimilado.

El cómo resolver esto es algo que no llegamos a solucionar más que repitiendo el proceso una y otra vez, en acciones diversas para que como el "rosa-rosae" o como se les dice "inglés se aprende hablando con un nativo, no sólo aprendiendo vocabulario". Al tratarse de un contenido procedimental es necesaria la práctica continuada para poder adquirir esos aprendizajes. 
Por conocimientos erróneos aprehendidos con anterioridad, suponen que el vincular (colocar) contenidos puede gestionarse con extensiones estándares conocidas por el público en general como jpg para las imágenes.

\section{Cuarta sesión}

Siguiendo el "método-mancera" en Illustrator se formulan estrategias para procesar convenientemente los contenidos gráficos en cualquier producto editorial.

En relación al viernes anterior se determinan distintos formatos y se identifican probabilidades y diferentes tamaños con que trabajar para procesar el contenido en las distintas propuestas que se han ido diseñando en InDesign. El alumnado al ver la resolución del gráfico, una vez colocado, es capaz de reconocer si está bien construido o no en Illustrator. Es ahora cuando muchos se dan de bruces con la realidad de lo que tienen delante y confirman que, si hubieran, en su momento, atajado un pequeño problema, ahora no estarían delante de un desastre que para arreglarlo deben dedicarle mucho tiempo.

Algo que sería conveniente para que puedan comprender con mejor acierto algunos parámetros en la calidad de la imagen, sería poder imprimir algunos referentes en el aula y así comprobar fisicamente, en lugar de conceptualmente (Porlán, R. 2017), el resultado en papel de ciertos gestores técnicos. Digitalmente, por otra parte, es viable reconocer el mismo proceso, aunque como la gestión de puntos en la imagen es muy diferente, es mejor poder identificarlo en ambos casos: digital y fisico.

\section{Quinta y sexta sesión}

A punto de finalizar el proceso editorial, más allá de haber procesado los estilos de párrafo y de carácter, enlaces de cajas de texto, texto fluido en páginas maestras, estilos de objeto, gestión de capas... nos centramos en un 
proceso complejo como es el uso de la tabla de contenidos, necesaria e indispensable para rematar un proyecto.

Excepto algunas dudas puntuales, tras haber asimilado todos los procesos anteriormente expuestos, el colectivo comprende y ha asimilado el desarrollo de un índice automático (tabla de contenido). Para verificar que esto es así, se insta a algunos que han dado por comprendido el método, para que sean quienes expliquen a otros compañeros, por grupos, cómo se ha de hacer. Para complicar la situación se les plantean algunos cambios (tanto desde el texto como desde la imagen), que sólo pueden ser subsanados si procesan intelectualmente el método-mancera y, para sorpresa general, está más que entendido ya que pueden, a expensas de una pequeña ayuda, desenvolverse por ellos mismos.

\section{Séptima y octava sesión}

Todo proyecto ha de ser salvaguardado convenientemente. Para ello se les indican los conceptos de empaquetar (guardar proyectos por carpetas ordenadas) y de exportar (para obtener un formato concreto a conveniencia). Tal y como se les planteaba en el cuestionario, esto es algo indispensable para dominar y controlar todo el trabajo desde el inicio al final.

En cuanto al proceso de exportar se plantean distintos niveles. Son los propios alumnos quienes proponen condiciones para saber cómo exportar y en qué soportes pueden ser vistos mejor: distintas plataformas, distintos procesadores o diferentes formatos multiplataforma.

Una vez que hemos planteado todo el proceso necesario para gestionar un proyecto editorial, se ve más fácilmente cómo una cosa hace que la otra requiera ser trabajada de un modo concreto, para que no de problemas futuros.

El interés suscitado en el procesado de datos y cómo guardar todo el contenido ha sido alto. Sobre todo, porque 
en un alto porcentaje todos tendrán que exportar su TFG para imprimir, exportarlo para presentarlo online y además deberán construir una presentación de este.

Este ciclo de mejora concluye pasando de nuevo el cuestionario que, por faltas de asistencia, de los 22 del grupo de mañana que lo hicieron inicialmente, tan sólo se podrán cotejar 15 finalmente; y de los 25 del grupo de tarde tan sólo 20 pudieron ser confrontados.

\section{Evaluación del aprendizaje de los estudiantes}

Una vez se recogieron y analizaron las primeras respuestas se pudo comprobar que las preguntas y sus distintos niveles de respuesta podían haberse formulado mejor. No obstante, como lo lógico, para analizar la evolución del conocimiento, era mantener las mismas preguntas, aquellas se mantuvieron. Se incluye el análisis por categoría de las respuestas del cuestionario inicial y final para la pregunta cuatro del cuestionario: ¿Qué aspectos debemos tener en cuenta para construir una maquetación?

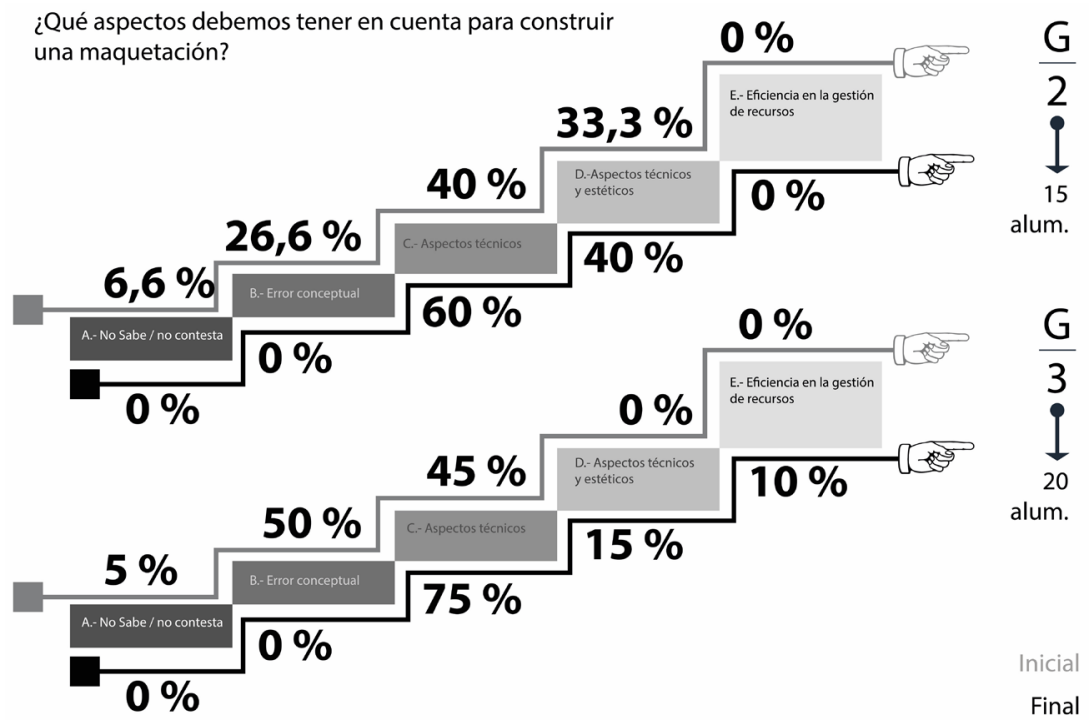

Figura 3. Escalera de aprendizaje de la pregunta cuatro del cuestionario.

Jornadas de Formación e Innovación Docente del Profesorado | № 2 (2019) Esta obra se distribuye con la licencia Creative Commons Reconocimiento-NoComercial-SinObraDerivada Internacional (CC BY-NC-ND 4.0.) 
Como se puede apreciar, se establecieron cinco niveles de respuesta para esta pregunta:

A.- No sabe / No contesta.

B.- Error conceptual. Equivocación de conceptos.

C.- Aspectos técnicos. El principal condicionante para producir una maqueta está en conocer la técnica y cómo emplearla.

D.- Aspectos técnicos y estéticos. A este dominio técnico se suma el planteamiento estético que engrandece el producto o le da una identidad propia

E.- Eficiencia en la gestión de recursos. Lo importante que el alumnado debe identificar es cómo trabajar las herramientas para procesar adecuadamente los distintos recursos.

Como valor de reconocimiento está el conseguir visualizar, en este modelo de evaluación continua que se gestiona en la asignatura, las respuestas que no se formulan en un examen. Además, se hace tangible la dificultad de comprensión e incluso los puntuales problemas de desconexión que se han registrado en algunos. Ante esta singularidad, sería conveniente consultar a título particular cómo tras dar una respuesta acertada se ha podido pasar a responder después con un nivel inferior.

Con independencia de estas puntualizaciones y teniendo en cuenta las 36 personas que completaron la primera y segunda encuesta podemos indicar que el 5\% subió directamente del nivel A al C. En torno al 38\% subieron del nivel B al C. El 25\% mantuvo el nivel C. El 13\% subió al nivel $D$ desde distintos niveles inferiores. Y el 5\% subió al nivel máximo.

Objetivamente, por tanto, se puede obtener un resultado positivo, de la asimilación de los datos, gracias a la aplicación del segundo ciclo de mejora. 
En próximos cursos, y para otras cuestiones del programa, lo que se va a tener en cuenta es reformular las preguntas de manera más ajustada y adaptar las actividades para que no queden lagunas de ningún tipo respecto a la comprensión de los medios técnicos y estéticos.

Más allá de todo esto, necesitamos cambiar, con independencia de seguir reforzando conceptos de forma personalizada, para mejorar el flujo de trabajo y ahondar en promover la resolución de problemas de manera colectiva (Morón Domínguez, 2015).

\section{Evaluación del CIMA}

\section{Cuestiones a mantener y cambios a introducir}

Con independencia de la satisfacción que pueda extraerse de los resultados de las escaleras y de buscar nuevos recursos para reforzar los conocimientos, son varios los puntos a considerar:

1. La teoría aplicada a la práctica (modelo asentado en las Bellas Artes) es esencial para mediante la praxis asentar y con ello edificar sobre la acción el proceso intelectual.

2. Involucrar al alumnado en la toma de decisiones (Vergara Ramírez, 2016) para crear los proyectos es un modo de hacer más dinámica la docencia y adaptarla a sus propias necesidades. Aunque puede resultar más agotador, finalmente, y sin dudarlo, es mucho más satisfactorio y enriquecedor para el colectivo.

3. Formular estos proyectos en base a una realidad (Swartz, Costa, Beyer, Reagan, Kallick 2016) es la esencia de todo este programa. Superar el concepto de ejercicio para construir un producto 
que les sirva para su proyección de futuro y controlar los procesos y métodos a seguir para profesionalizar en un futuro sus trabajos es algo que se considera necesario.

Entendiendo la satisfacción general obtenida en el colectivo, tanto por parte del alumnado al obtener productos finales de calidad, como por parte del docente por reconocer el empoderamiento del alumnado como un bien personal y común, todo es mejorable.

Por ello en futuras incursiones, en la sutil, pero real, consideración de que, al menos la siembra al final da su fruto, se sustenta una evaluación ponderada que intenta ver más allá de lo que es la fría valoración del proceso cognitivo. Las secuelas que, por abundamiento, se quedan ancladas en nosotros (alumnos de la vida) son las que dan valor a la circunstancia de saber que, queriendo alcanzar la meta, siempre se busca y persigue algo mejor que nos haga crecer por y para los demás. Esa esencia es la que, por ende, hay que localizar y propagar en todos aquellos que podamos y tengamos la posibilidad de inculcar.

Jornadas de Formación e Innovación Docente del Profesorado | № 2 (2019) Esta obra se distribuye con la licencia Creative Commons 


\section{Referencias bibliográficas}

Gómez-Aguilar, A. (2018) Ciclo de mejora docente para la asignatura de diseño y aplicaciones multimedia del grado en comunicación audiovisual. En R. Porlán y E. Navarro (coord.) Comunicación presentada en las Jornadas de Formación e Innovación Docente del Profesorado: JFIDOP 2018, 20 y 21 de diciembre. Sevilla: Universidad de Sevilla, pp. 1016-1034. Recuperado de http:/ / institucional.us.es/revistas/JDU/Gomez_Aguilar_Antonio.pdf

Morón Domínguez, C. (2015) La mejora de la práctica docente a través de la metodología de proyectos de investigación: El caso del profesorado de Andalucía del Proyecto Roma (Tesis doctoral) Universidad de Málaga, Facultad de Ciencias de la Educación. España. Recuperado de https://riuma.uma.es/xmlui/bitstream/ handle/10630/13129/TD_MORON_DOMINGUEZ_Alfonso_Carlos.pdf?sequence-1\&isAllowed=y

Porlán, R. (coord.) (2017) Enseñanza Universitaria. Cómo mejorarla. Madrid: Morata.

Swartz, R.J., Costa A. L., Beyer, B. K., Reagan, R., y Kallick, B. (2016) El aprendizaje basado en el pensamiento. Madrid: Ediciones SM. Colección: Biblioteca Innovación Educativa.

Vergara Ramírez, J. J. (2016) Aprendo porque quiero. El Aprendizaje Basado en Proyectos (ABP) paso a paso. Madrid: Ediciones SM. Colección: Biblioteca Innovación Educativa. 